\title{
III-V-on-silicon photonic integrated circuits for communication and sensing applications
}

\author{
G. Roelkens, A. Abassi, S. Keyvaninia, S. Uvin, K. Van Gasse, \\ Z. Wang, U. Dave, B. Kuyken, G. Morthier, D. Van Thourhout \\ Photonics Research Group, Ghent University/IMEC, Belgium \\ gunther.roelkens@intec.ugent.be
}

\begin{abstract}
We review the integration of III-V semiconductors on silicon photonic integrated circuits as a way of realizing fully integrated silicon photonic transceivers and short-wave infrared spectroscopic sensors.
\end{abstract}

Keywords-silicon transceivers; spectroscopic sensors

\section{INTRODUCTION}

Silicon photonics is emerging as an important technology for the realization of high aggregate bitrate transceivers. This is mainly driven by the fact that CMOS fabrication technology can be used to realize these photonic integrated circuits, resulting in cost reduction when manufactured in high volume. Also, the dense integration with silicon electronics offers the potential to substantially reduce the power consumption. The current silicon photonics technology offering comprises low loss waveguide circuits, high efficiency fiber-to-chip coupling structures and high performance silicon modulators and germanium p-i-n photodiodes operating at $28 \mathrm{Gbps}$ and beyond. The laser source integration has so far mostly relied on flipchip integration of III-V semiconductor devices, which is not a scalable approach and adds substantial cost to the transceiver fabrication. While silicon photonics development is mainly driven by optical interconnect applications, other application areas are also emerging. Especially in the context of sensing silicon photonics is of interest, due to the potentially low cost of such devices. Spectroscopic sensors, measuring the absorption signature of a sample of interest, stand out in terms of selectivity and allow real-time, calibration-free operation. As high-performance silicon-on-insulator waveguide circuits can be realized operating in the short-wave infrared (SWIR) wavelength range $(1-3 \mu \mathrm{m})$, one can conceive fully integrated SWIR spectroscopic sensors operating on this waveguide platform. However, this requires the integration of III-V laser sources operating in that wavelength range. In this paper we will discuss our work on III-V on silicon integration, on one hand to allow for a scalable laser integration approach for optical transceivers, and on the other hand to realize integrated spectroscopic sensors.

\section{III-V ON SILICON PHOTONIC INTEGRATED CIRCUITS FOR COMMUNICATION APPLICATIONS}

\section{A. Directly modulated III-V-on-silicon DFB lasers}

The standard single wavelength laser structure is a distributed feedback laser, comprising a first order feedback grating and a quarter wave shift. While such devices can be realized on a III$\mathrm{V}$ platfom, they require e-beam definition of the grating, facet coating and spotsize converters to efficiently interface with silicon photonic integrated circuits. Using heterogeneous integration - using DVS-BCB as the adhesive bonding agentDFB laser arrays can easily be integrated on silicon, in which case the grating structures can be defined in the silicon waveguide layer, no facet coatings are required since a lowreflection spot size converter can be integrated for the interfacing with the silicon waveguide circuit, as shown in Figure 1. Also, as the processing of the laser takes places after integration of the III-V onto the silicon waveguide circuit, no costly active alignment between laser and photonic IC is required [1].
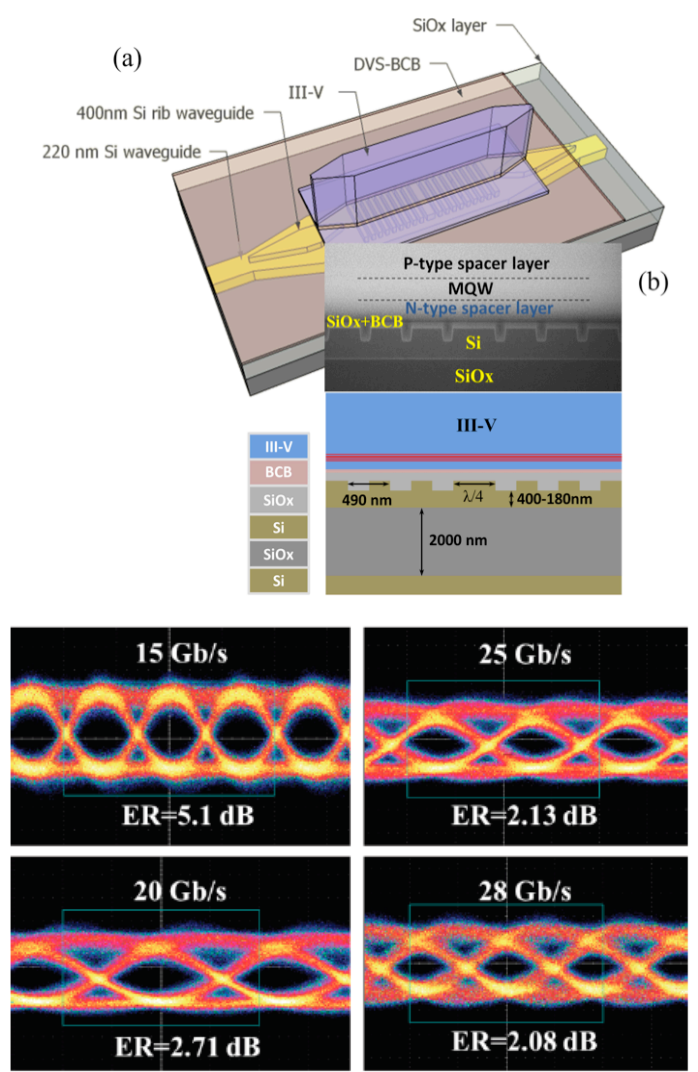

Figure 1: (a) layout of III-V on silicon DFB laser with the first order grating defined in the silicon waveguide layer; (b) SEM cross-section of the III-V-on-silicon laser structure. (c) direct modulation eye diagrams $(1.5 \mathrm{Vpp})$. 
$14 \mathrm{~mW}$ output power at room temperature, 9\% wall plug efficiency and a side mode suppression better than $50 \mathrm{~dB}$ are experimentally obtained [1]. Using direct modulation, 25Gbit/s transmission has been demonstrated over $1 \mathrm{~km}$.

\section{B. III-V-on-silicon mode-locked lasers}

In order to realize high aggregate bit rate transmitters optical time division multiplexing techniques can be implemented, using a short-pulse III-V on silicon mode-locked laser source. High performance lasers can be realized because of the low linear and nonlinear losses of silicon waveguides. Classical colliding pulse devices have been implemented including ring-cavity and Fabry-Perot cavity arrangements [2]. On the III-V-on-silicon platform we also implemented an anticolliding pulse type mode-locked laser structure as shown in Fig. 2, of which theory predicts to result in higher output power and lower timing jitter. In this case the saturable absorber is implemented on the low reflectivity output coupler, which is implemented as a III-V-on-silicon grating as shown Fig. 2. The high reflectivity grating is implemented as a strong DBR grating in the silicon waveguide layer. Passive mode-locking at $4.83 \mathrm{GHz}$ repetition rate generating 3 ps pulses is demonstrated. Over $9 \mathrm{~mW}$ waveguide coupled output power is obtained.

(a)

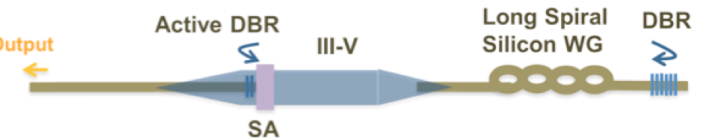

(b)

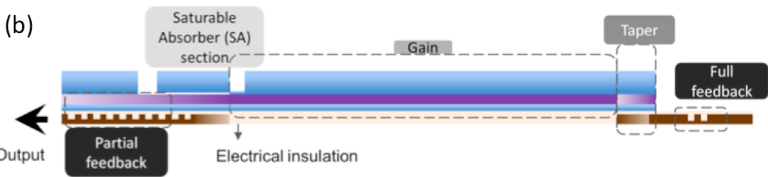

(c)

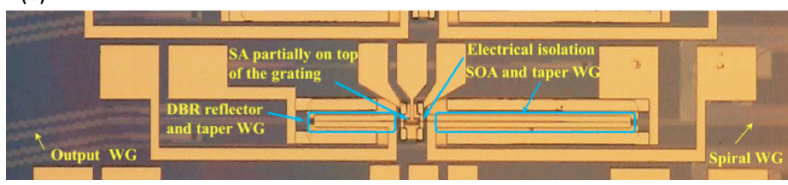

Figure 2: anti-colliding pulse type mode-locked laser geometry (a); longitudinal cross-section indicating the SOA and SA sections (b) and the generated optical output spectrum (c).

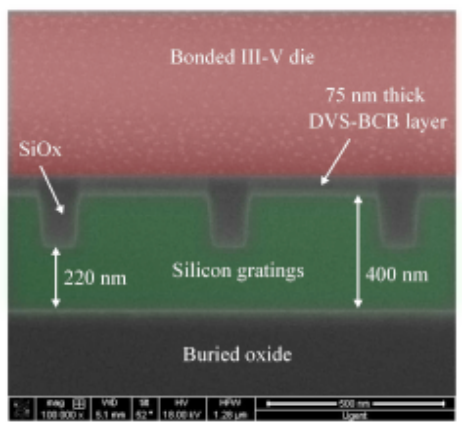

Figure 3: SEM picture of the III-V/ silicon bond interface at the modelocked laser saturable absorber section

\section{III-V ON SILICON PHOTONIC INTEGRATED CIRCUITS FOR SENSING APPLICATIONS}

In order to expand the application range of silicon photonics beyond optical communications, III-V on silicon integration can also be applied for spectroscopic sensor devices. Especially in the short-wave infrared high performance silicon photonic integrated circuits have been demonstrated. While the integration of long-wavelength lasers ( $>2 \mu \mathrm{m}$ wavelength) is pursued $[3,4]$, an alternative approach is to exploit the high peak power of the demonstrated $1.55 \mu \mathrm{m}$ III-V on silicon mode-locked lasers to realize a short-wave-infrared supercontinuum. While silicon waveguides allow strong optical confinement and have a high Kerr nonlinearity, the efficiency of the supercontinuum generation is hampered by parasitic two-photon absorption (TPA) in silicon at telecom wavelengths. Therefore we have been focusing on supercontinuum generation in wide bandgap III-V semiconductor photonic wire waveguides integrated on a silicon substrate. InGaP is used as the waveguide material as it shows no TPA at $1.55 \mu \mathrm{m}$ operation and also has a strong Kerr nonlinearity. Octave spanning supercontinuum generation was demonstrated in a dispersion engineered $\mathrm{InGaP}$ on silicon waveguide as shown in Fig. 3, thereby paving the way towards wide wavelength coverage spectroscopic sensors in the shortwave infrared.

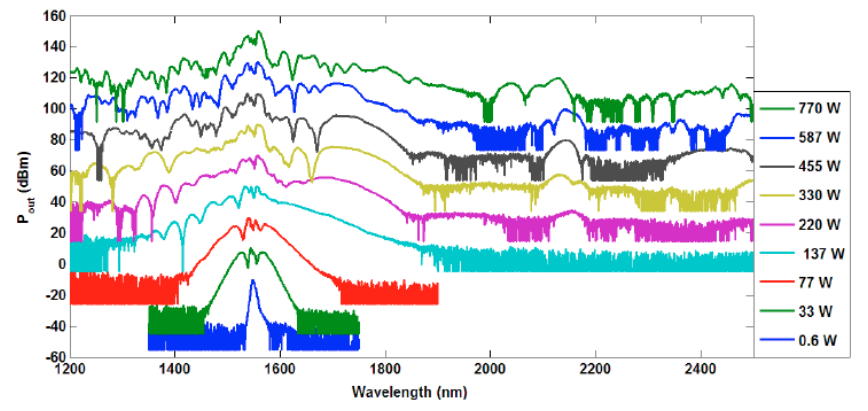

Figure 3: InGaP on Si photonic wire waveguide for short-wave infrared supercontinuum generation

[1] S. Keyvaninia, S. Verstuyft, L. Van Landschoot, D. Van Thourhout, G. Roelkens, G. Duan, F. Lelarge, J.M. Fedeli, S. Messaoudene, T. De Vries, B. Smalbrugge, E.J. Geluk, J. Bolk, M.Smit, Heterogeneously integrated III-V/silicon distributed feedback lasers, Optics Letters, 38(24), p.5434-5437 (2013)

[2] S. Keyvaninia, S. Uvin, M. Tassaert, X. Fu, S. Latkowski, J. Marien, L. Thomassen, F. Lelarge, G. Duan, P. Verheyen, G. Lepage, J. Van Campenhout, E. Bente, G. Roelkens, Narrow-linewidth short-pulse III$\mathrm{V}$-on-silicon mode-locked lasers based on a linear and ring cavity geometry, Optics Express, 23(3), p.3221-3229 (2015)

[3] G. Roelkens, U.D. Dave, A. Gassenq, N. Hattasan, C. Hu, B. Kuyken, F. Leo, A. Malik, M. Muneeb, E.M.P. Ryckeboer, D. Sanchez, S. Uvin, Z. Hens, R. Baets, Y. Shimura, F. Gencarelli, B. Vincent, R. Loo, J. Van Campenhout, L. Cerutti, J.B. Rodriguez, E. Tournie, X. Chen, M. Nedeljkovic, Silicon-based photonic integration beyond the telecommunication wavelength range, IEEE Journal of Selected Topics in Quantum Electronics (invited), 20(4), p.8201511 (2014)

[4] A. Spott, M. Davenport, J. peters, J. Bovington, M. Heck, E. Stanton, I. Vurgaftman, J. Meyer, J. Bowers, heterogeneously integrated 2.0um CW hybrid silicon lasers at room temperature, Optics Letters 40(7), p.14801483 (2015) 